estas terapias no toman en cuenta los problemas físicos que enfrentan estos pacientes, como la fatiga, la pérdida de capacidad funcional y el aumento de peso. Los ejercicios pueden mejorar una amplia gama de problemas que afectan a la calidad de vida después del diagnóstico de cáncer. Un estudio observacional prospectivo de sobrevivientes de cáncer de mama indicó que el riesgo de muerte se redujo en $50 \%$ en las mujeres que se mantenían activas en comparación con las que permanecieron inactivas después del diagnóstico. Una revisión sistemática reciente sobre el efecto de los ejercicios en las pacientes con cáncer de mama y sobrevivientes de esa enfermedad concluyó que los ejercicios mejoraron la calidad de vida, la capacidad cardiorrespiratoria y el funcionamiento físico y redujeron la fatiga.

En este estudio se evaluó si la participación en un programa supervisado de ejercicios en grupo para mujeres en tratamiento por cáncer de mama en sus primeros estadios ofreció beneficios funcionales y psicológicos durante el tratamiento y si esos beneficios se mantuvieron seis meses después de la intervención.

Se captaron 203 pacientes con cáncer de mama en estadios tempranos (0-III) durante la sesión de asignación de quimioterapia o radioterapia ambulatorias en tres centros oncológicos pertenecientes a los Servicios Nacionales de Salud, en Escocia, Reino Unido. Se excluyeron las pacientes con hipertensión no controlada, enfermedades respiratorias, disfunción cognitiva o que realizaban ejercicios con regularidad. Las pacientes se asignaron aleatoriamente a un grupo de ejercicios o a otro de control. La evaluación se realizó al inicio, a las 12 semanas y a los 6 meses (117 pacientes completaron el seguimiento).

La calidad de vida se estableció mediante la evaluación del tratamiento contra el cáncer (físico, funcional, social y emocional), el nivel de fatiga y los síntomas endocrinos. Como indicador secundario de los resultados se empleó el inventario de depresión de Beck, la escala de afectos positivos y negativos, el índice de masa corporal, el recuento de las actividades físicas en los últimos siete días, el desempeño en la prueba de caminar 12 minutos y la puntuación en la prueba de movilidad de los hombros.

Después de 12 semanas de ejercicios supervisados, el grupo de intervención mostró beneficios en el funcionamiento físico y psicológico en comparación con el grupo de control. No se informaron efectos adversos. Los beneficios informados a las 12 semanas por el grupo que participó en el programa de ejercicios se mantuvieron a los seis meses de seguimiento, con la excepción del tiempo autoinformado de ejercicios físicos. Los beneficios específicos relacionados con la calidad de vida y el cáncer de mama se hicieron evidentes solo a los seis meses, cuando la mayoría de las pacientes habían concluido el tratamiento.

No se logró identificar qué parte del programa de ejercicios generó mayores beneficios. Los datos cualitativos indican que probablemente el efecto de grupo mismo constituyó un aspecto importante. Además, las mejorías en las pruebas de la caminata de 12 minutos y en la de la movilidad de los hombros en el grupo de intervención fueron más atribuibles a los ejercicios en sí que al efecto de grupo.

De esta manera, se concluye que los ejercicios supervisados en grupo, sumados a la atención médica habitual que reciben las mujeres tratadas por cáncer de mama en sus estadios tempranos, ofrecieron beneficios funcionales y psicológicos adicionales. Los médicos deben recomendar a sus pacientes con cáncer de mama mantenerse en actividad y los encargados de diseñar las políticas sanitarias deben ofrecer la posibilidad de practicar ejercicios físicos en los servicios de rehabilitación de pacientes con cáncer, de manera similar a los que se ofrecen para la rehabilitación cardíaca. Se requieren investigaciones adicionales para determinar el efecto de los ejercicios en pacientes con otros tipos de cáncer. (Mutrie N, Campbell AM, Whyte F, McConnachie A, Emslie C, Lee L, et al. Benefits of supervised group exercise programme for women being treated for early stage breast cancer: pragmatic randomised controlled trial. Br Med J. 2007;334:517-22.)

\section{Reducción de la mortalidad infantil en Chile: un éxito en dos etapas}

La mortalidad infantil ha disminuido en la Región de las Américas de 36,9 por 1000 nacidos vivos en el quinquenio 1980-1985 hasta 24,8 por 1000 nacidos vivos en el período 1995-2000. Esta mortalidad, no obstante, se distribuye de forma desigual: mientras en algunos países - como Chile, Costa Rica y Cuba - las tasas están cercanas o por debajo de 10 por 1000 nacidos vivos, en otros - como Bolivia y Haití - se mantienen por encima de 50 por 1000 nacidos vivos. El elemento común en los casos más exitosos ha sido la fuerte intervención estatal, mediante políticas sanitarias estructuradas y sostenidas —enfocadas hacia la salud materno-infantil y el entorno del hogar- y otras políticas sociales relevantes, como la universalización de la educación básica.

En el caso de Chile se observan dos claras etapas en la reducción de la mortalidad infantil. La primera etapa comenzó en la década de 1950, cuando la tasa de mortalidad infantil era de 136,2 por 1000 nacidos vivos (con 29,0 debido a enfermedades infecciosas, diarreas y deshidratación; 44,4 por enfer- 
medades respiratorias y 38,7 por problemas relacionados con las condiciones perinatales). En 1952 se estableció el Servicio Nacional de Salud (SNS), mediante la fusión de varias instituciones y organizaciones que formaban parte de un complejo pero exitoso proceso de reforma. El principal foco de acción de ese sistema de salud pública fue la salud materna e infantil.

En Chile, las intervenciones relacionadas con la salud de la madre y el niño se comenzaron a aplicar conjuntamente con el desarrollo de una atención sanitaria basada en criterios científicos y con un enfoque sistémico. Estos criterios eran: 1) una visión integrada de la salud y el ciclo de vida con el medio ambiente, que daba preferencia a la atención sanitaria ambulatoria y en la comunidad; 2) un concepto integrado de la atención sanitaria y las organizaciones sanitarias, en el que cada acción era parte de una estrategia holística; 3) equipos multidisciplinarios de atención sanitaria, con profesionales de diversas especialidades que incorporaban diferentes habilidades y funciones complementarias; 4) acciones de entrenamiento e investigación, mediante la integración de los servicios de salud pública con las universidades; 5) evaluación continua de los programas e instrumentos; y 6) mejoramiento permanente de la calidad y fiabilidad de los datos epidemiológicos - entre ellos, de la certificación médica de las causas de muerte- y el análisis de las muertes infantiles.

Para 1990, la mortalidad infantil había bajado a 16 por 1000 nacidos vivos (una reducción de $88 \%$ ), gracias al mejoramiento de las condiciones perinatales (mortalidad de 5,5 por 1 000; reducción de $35 \%)$, la disminución de nacimientos con malformaciones congénitas $(3,7$ por $1000 ; 23 \%)$ y de infecciones respiratorias (2,4 por $1000 ; 16 \%)$.

La segunda etapa en la reducción de la mortalidad infantil estuvo más ligada a las intervenciones de salud que al mejoramiento de las condiciones socioeconómicas. En el año 2000, la mortalidad infantil bajó a 8,9 por 1000 nacidos vivos, en primer lugar gracias a la disminución de la mortalidad por infecciones respiratorias (de 2,4 a 0,66 por 1000 ); el mejoramiento de las condiciones perinatales (de 5,5 a 3,4 por 1000$)$ y al control de las malformaciones congénitas (de 3,7 a 3,0 por 1000 ).

El presupuesto total destinado a la salud en Chile en el año 2000 equivalía a \$US 2,28 mil millones, de ellos solo $\$$ US 16,75 millones correspondieron a los cuatro programas nuevos o rediseñados de salud materna e infantil, es decir, una fracción mínima del presupuesto de salud. Si se toma en cuenta que gracias a la reducción de la mortalidad infantil sobreviven 285 niños más anualmente, el costo de salvar cada vida sería el equivalente de \$US 58777,00 .

A pesar de que las experiencias implementadas en Chile tienen diferentes niveles de eficacia y deben estudiarse con mayor profundidad, los resultados alcanzados demuestran que se puede reducir la mortalidad infantil mediante mejoras sociales y programas específicos de salud pública. (Jiménez J, Romero MI. Reducing infant mortality in Chile: success in two phases. Health Aff. 2007;26(2):458-65.)

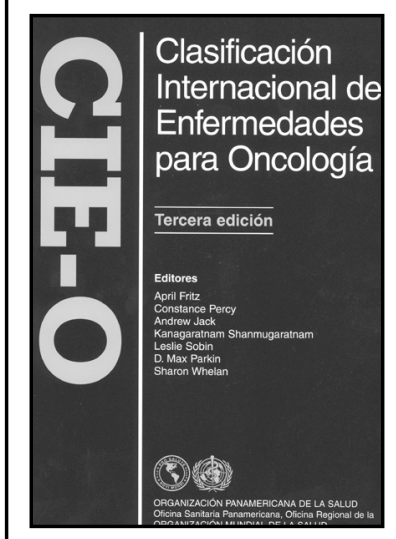

\section{Clasificación Internacional de Enfermedades para Oncología (CIE-O) 3. ${ }^{a}$ ed.}

La Clasificación Internacional de Enfermedades para Oncología (CIE-0) se ha usado durante casi 25 años como un instrumento estándar para codificar los diagnósticos de las neoplasias en los registros de tumores y de cáncer y en los laboratorios de anatomía patológica.

La CIE-0 es una clasificación dual con sistemas de codificación tanto para la topografía como para la morfología. El código topográfico describe el sitio de origen de la neoplasia y usa las mismas categorías de tres y cuatro caracteres que la sección de neoplasias del capítulo II de la CIE-O. El código morfológico describe las características del tumor mismo, incluidos su tipo de células y su actividad biológica. El índice alfabético presenta los códigos para la topografía y la morfología, e incluye aquellas lesiones y trastornos que se asemejan a los tumores.

En la última sección se presenta una guía para reconocer las diferencias de la codificación morfológica entre la segunda y la tercera edición. La guía comprende las listas de todos los códigos nuevos, los términos incor-

$2002 \cdot 320 \mathrm{pp}$.

ISBN 9275315868

Código: PC 586

Precio: US\$ 25.00 en América Latina y el Caribe/ US $\$ 34.00$ en el resto del mundo porados y los sinónimos agregados a las definiciones de los códigos existentes, la definición de los términos nuevos en los códigos de morfología, los términos que designan los trastornos que actualmente se consideran malignos, los términos eliminados y los términos que cambiaron en los códigos de comportamiento.

Adquiera esta publicación por medio de la librería en línea de laOPS: http://publications.paho.org Fax: (301) 206-978•Correo electrónico: paho@pmds.com•OPS/OMS oficina de país 